\title{
Current status and new horizons in Monte Carlo simulation of X-ray CT scanners
}

\author{
Habib Zaidi · Mohammad Reza Ay
}

Received: 18 December 2006/Accepted: 2 June 2007/Published online: 5 July 2007

(C) International Federation for Medical and Biological Engineering 2007

\begin{abstract}
With the advent of powerful computers and parallel processing including Grid technology, the use of Monte Carlo (MC) techniques for radiation transport simulation has become the most popular method for modeling radiological imaging systems and particularly X-ray computed tomography (CT). The stochastic nature of involved processes such as $\mathrm{X}$-ray photons generation, interaction with matter and detection makes MC the ideal tool for accurate modeling. MC calculations can be used to assess the impact of different physical design parameters on overall scanner performance, clinical image quality and absorbed dose assessment in CT examinations, which can be difficult or even impossible to estimate by experimental measurements and theoretical analysis. Simulations can also be used to develop and assess correction methods and reconstruction algorithms aiming at improving image quality and quantitative procedures. This paper focuses mainly on recent developments and future trends in X-ray CT MC modeling tools and their areas of application. An overview of existing programs and their useful features will be given together with recent developments in the design of computational anthropomorphic models of the human anatomy. It should be noted that due to limited space, the references contained herein are for illustrative purposes and
\end{abstract}

H. Zaidi $(\bowtie) \cdot$ M. R. Ay

Division of Nuclear Medicine, Geneva University Hospital,

1211 Geneva 4, Switzerland

e-mail: habib.zaidi@hcuge.ch

Present Address:

M. R. Ay

Department of Medical Physics and Biomedical Engineering, School of Medicine, Medical Sciences/University of Tehran,

Tehran, Iran are not inclusive; no implication that those chosen are better than others not mentioned is intended.

Keywords X-ray computed tomography (CT) . Monte Carlo simulations - Detector - Scatter . Computational models

\section{Introduction}

X-ray computed tomography (CT) is a medical imaging modality that produces cross-sectional images representing the X-ray attenuation properties of the body $[1,2]$. Unlike conventional two-dimensional (2D) projection imaging, CT does not suffer from interference from structures in the patient outside the slice being imaged. Recent developments in X-ray CT instrumentation spurred the development of multi-slice and flat-panel detector configurations thus offering significant improvements in volume coverage, isotropic spatial resolution and contrast utilization [3]. Despite worthwhile research efforts and achievements in instrumentation, X-ray CT images still have the inherent tendency to produce physics-related artifacts owing to the fact that the images are reconstructed from a large number of independent detector elements. There are several sources of error and artifact that affect both clinical and small animal X-ray CT image quality [4, 5]. The assessment of their relevance is generally commended with the aim to reduce their impact either by optimizing the scanner design or by devising appropriate image correction and reconstruction strategies.

The evaluation of the effect of physical, geometrical and other design parameters on scanner performance and resulting image quality and patient dose could be achieved through cumbersome experimental measurements using 
developed test prototypes [6, 7] or more conveniently using deterministic mathematical modeling [8-10] or sophisticated Monte Carlo (MC) simulations [11, 12]. The latter relies on either in-house developed dedicated programs [5, $11,13,14]$ or public domain general purpose MC codes such as MCNP [12], EGS4 [15] and GEANT4 [16]. Nowadays, the MC method is widely used for solving many scientific problems involving statistical processes and is particularly well suited for medical physics and biomedical engineering applications due to the stochastic nature of radiation emission, transport and detection processes. The general idea of MC analysis is to create a model as similar as possible to the real system under study and calculate the interaction within the modeled system based on known probabilities of occurrence using random sampling of probability density functions for each event. An overview of the fundamentals of MC techniques and their applications in diagnostic medical imaging and radiation dosimetry can be found in [17-19]. The computational burden of $\mathrm{MC}$ calculations compared to fast analytical modeling remains the bottleneck for their introduction in clinical environments. However, the widespread availability of high performance parallel computing and more recently Grid technology in addition to the popularity of variance reduction techniques spurred their development and application to a wide variety of problems particularly when modeling complex geometries.

\section{Dedicated simulators for modeling of X-ray CT scanners}

The simulation of X-ray CT imaging to assess qualitatively and quantitatively the image formation process and interpretation and to assist development of new detector configurations using deterministic methods and simplifying approximations has been developed mainly to improve speed of operation $[3,10]$. Analytical simulators are generally used for generation of raw data sets to evaluate correction and reconstruction algorithms [20], whereas MC-based simulators can, in addition to the above nominated purposes, be used for more realistic in-depth assessment of the effect of different design parameters on system performance and resulting image quality. Moreover, they offer the possibility to estimate physical parameters (e.g., scatter) that are difficult or even impossible to calculate using experimental measurements and analytical modeling. Analytical X-ray CT simulators are based on projection ray-tracing methods for the threedimensional (3D) calculation of intersections between trajectories of photons emitted from the X-ray tube focal spot toward the detector elements and all voxels or surfaces' equations for each $\mathrm{X}$-ray energy bin since the attenuation coefficients of different materials are energy-dependent. One such example is the simulator developed by De Man [20] for single-slice detector configurations using the IDL language (Research Systems Inc., Boulder, CO) with the aim to evaluate his developed iterative reconstruction algorithm for reduction of metallic artifacts in X-ray CT. The effect of different sources of error such as beam hardening, partial volume effect, scattered radiation, motion and aliasing has been theoretically considered in this simulator. Another example is a CT simulator specifically designed to simulate dynamic cardio-pulmonary studies with single- and multi-slice detector CT (MDCT) operating in spiral mode to optimize the performance of reconstruction algorithms in dynamic studies [21].

MC-based simulations are based on direct transport of photons and electrons inside the materials in a 3D geometry. Their use for accurate dosimetry calculations in X-ray CT scanning is well established [15, 22], however, since the X-ray CT detection system is not modeled for such applications, the assessment of imaging related factors is not possible. Some MC simulators were developed for simulating conventional radiographic $\mathrm{X}$-ray imaging chain [23-25]. Though, to the best of our knowledge, there exist only three dedicated MC-based X-ray CT simulators, which can be used for both imaging and dosimetry modeling purposes. Colijn et al. [11] developed a rapid MCbased micro-CT simulator, refereed to here as accelerated MC simulator (AMCS), dedicated for modeling the SkyScan 1076 (SkyScan, Aartselaar, Belgium) cone-beam small-animal X-ray CT scanner. The simulator has been validated through comparison with experimental measurements of water phantom with various diameters, some containing steel and Teflon rods. The simulation process is divided in two main parts: the projection data of primary $\mathrm{X}$-ray photons are computed with a ray-tracer and the scatter distribution is estimated using an accelerated MC simulation method [5]. More recently, Ay and Zaidi [12] developed a MC-based X-ray CT simulator for fan- and cone-beam geometries with single-slice, multi-slice (up to 64 slices) and flat-panel detector configurations based on the general purpose MCNP4C radiation transport computer code. The simulator was validated through comparison with experimental measurements of different nonuniform phantoms with various sizes using both a clinical GE HiSpeed X/iF (General Electric Healthcare Technologies, Waukesha, WI, USA) fan-beam X-ray CT scanner and a small-animal SkyScan 1076 cone-beam X-ray CT scanner. A graphical user interface (GUI) running under Matlab 6.5.1 (The MathWorks Inc., Natick, MA, USA) creates the geometry of the scanners in different views as MCNP4C input file based on user selected design parameters. The simulator includes the possibility of detailed simulation of the X-ray tube [26], collimator, bow-tie filter, phantom, 

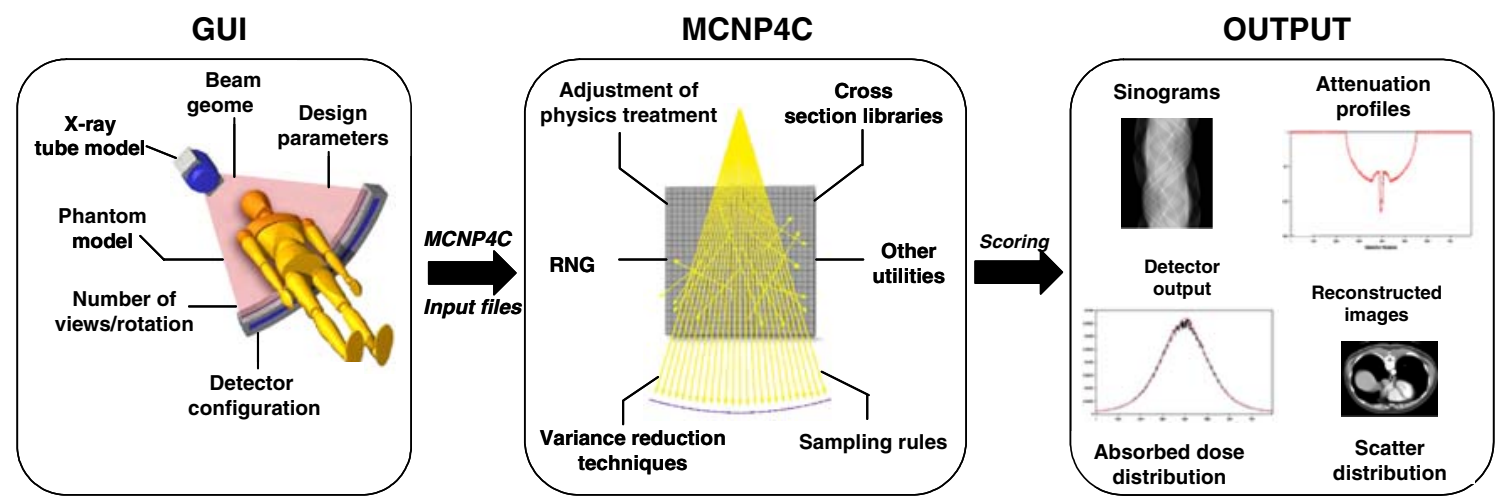

Fig. 1 Principles and main components of the MCNP4C-based Monte Carlo program dedicated for simulation of X-ray CT imaging systems

detector geometry and material. Figure 1 illustrates the principles and main components of the MCNP4C-based Monte Carlo simulator as applied to model an X-ray CT imaging system. The third MC code called CTmod, uses MC transport of photons inside simple phantoms for a single row of detector elements and has been used mainly for scatter modeling in the cone-beam geometry [14]. Unfortunately, only few details about this program at the time of writing this review are available as the authors are still refining the code and working on validation issues that will likely be covered in future publications by this group.

It should be emphasized that as opposed to radiation dosimetry where many studies investigated potential differences between the results obtained using different computational Monte Carlo codes [27], very few studies addressed this issue in simulation of medical imaging systems. Fig. 2 shows the comparison of measured and simulated profiles from water phantom containing Teflon and steel rods presented in log-linear scale in order to magnify the differences between simulated and measured results. It appears that MCNP4C has better agreement with the measured profile compared to AMCS (Figure 2b). The high absolute normalized error in the area covered by steel rods is due to the high attenuation of steel and probably small geometrical misalignment between simulated and experimental setups. The comparison between calculated SPR further illustrates the discrepancy between AMCS and MCNP4C in the region corresponding to steel rod location for the reasons discussed above (Fig. 2c).

\section{Applications of Monte Carlo simulations in CT}

The application of MC modeling in X-ray CT imaging research is an everlasting enthusiastic topic and still is an area of considerable interest. Today's applications of MC techniques in the field of radiological imaging include performance assessment and optimization of design geometries and scanning parameters [12], scatter charac- terization and rejection strategies [5, 13, 14], search for ideal detector configuration and material [16], generation of data sets for testing reconstruction and beam hardening correction algorithms and absorbed dose calculations to assess radiobiological risk from CT scans.

Although CT is a diagnostic imaging modality delivering higher patient dose in comparison with other diagnostic radiological procedures, its excellent spatial resolution stimulated its use in diagnostic imaging. In addition, the introduction of MDCT with its greater axial coverage and faster gantry rotation times increased the diagnostic utility of CT scanning. To optimize patients' selection, scanning protocols and benefit-to-risk ratios, substantial efforts were made by radiation protection committees to estimate radiation dose to patient for various CT examinations [28]. Given that the absorbed dose depends on various scanning parameters such as $\mathrm{mA}, \mathrm{kVp}$, pitch, slice thickness ...etc., the Monte Carlo method was adopted as the most convenient and powerful tool for organ and effective dose assessment [19]. The accuracy of MC calculations is well established for both axial [15] and spiral [6, 22, 29-34] scanning modes. One of the predominant methods for assessment of organ absorbed dose in CT examinations is the application of conversion coefficients derived by Monte Carlo calculations. CTDOSE is one of the earliest codes allowing calculation of organ and effective dose in diagnostic CT procedures [35]. Currently, CTDosimetry is the most popular dose calculation software, which makes use of the former National Radiological Protection Board (NRPB) Monte Carlo dose data sets published in its report SR250 [36]. This package allows to calculate patient organ and effective doses for the majority of commercially available CT scanners including most recent CT scanners with 64 slice capability [37].

With the advent of multiple-row and flat-panel detector configurations in addition to the slip-ring technology, there have been rapid developments in the design of clinical and small-animal CT scanners including X-ray tube specifications, geometrical magnification, detector configuration 

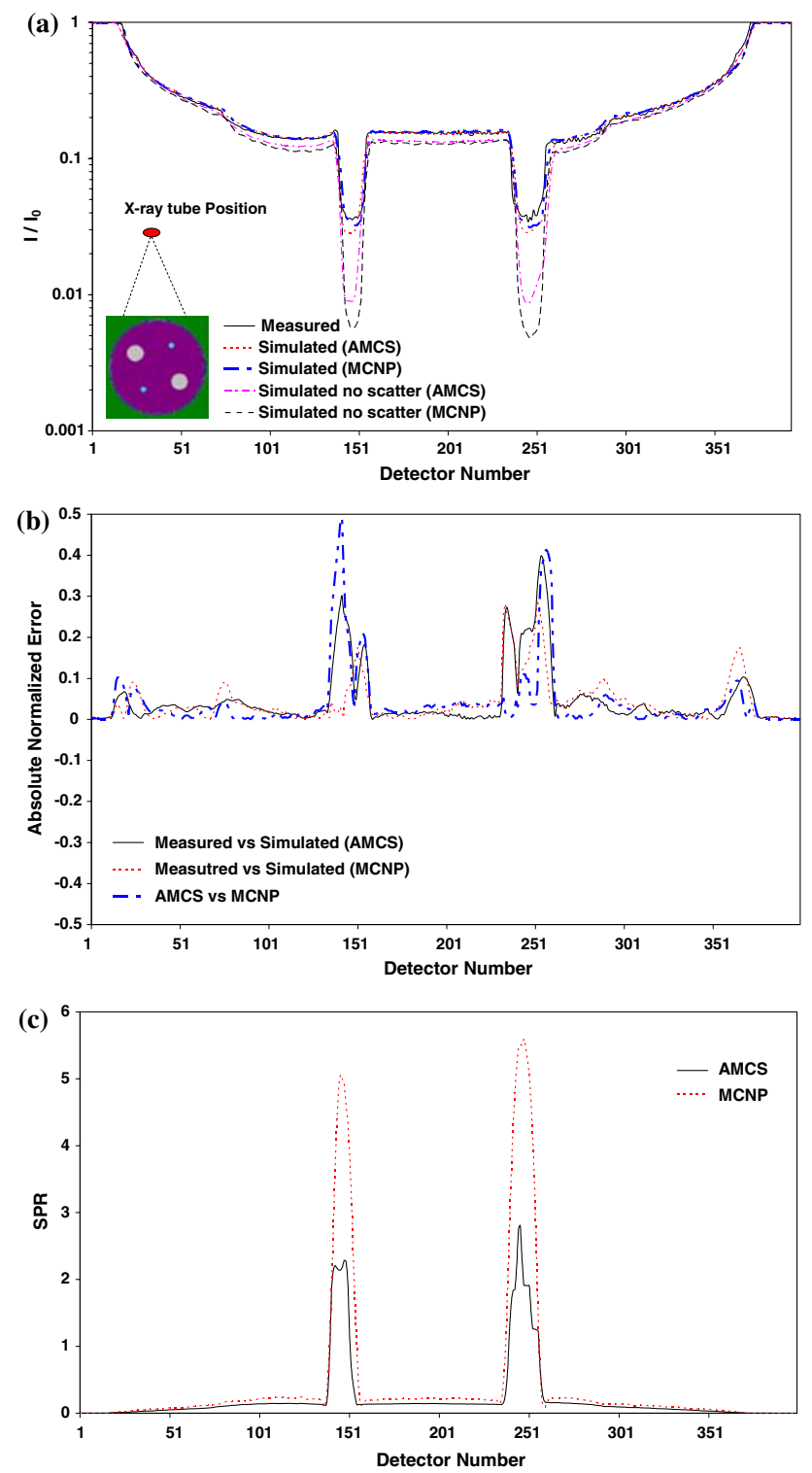

Fig. 2 Comparison between MCNP and AMCS simulated profiles and experimental measurements from water phantom containing steel and Teflon rod inserts [11] using the SkyScan small-animal X-ray CT scanner at tube voltage $100 \mathrm{kVp}$ showing (a) total and primary profiles; $\mathbf{b}$ the absolute normalized error of the profiles shown in a; and $\mathbf{c}$ the scatter to primary ratio for the simulated data. Reprinted with permission from [12]

and dose management. Ay et al. [26] investigated the effect of different parameters such as target material, target/filter combinations, voltage ripple, anode heel effect and focal spot size on the generated X-ray spectra using MC simulations. The impact of target angle on off-axis spectra, which is an important factor for covering the whole detector aperture by the X-ray beam in CT particularly for multi-slice systems, was also investigated. Although different analytical computational models with varying degrees of accuracy can be used for generation of X-ray spectra [38], MC simulations offer many additional advantages including the possibility of optimizing tube design and developing new target/filter combinations to improve image quality and reduce patient dose. The optimization of geometrical magnification in X-ray $\mathrm{CT}$, which depends on the source to detector and iso-center distances, has been investigated using theoretical calculations [3, 9]. The optimal detector's element material and size, which depend on the balance between image resolution, patient dose and signal to noise ratio, is another active research area where MC modeling plays an important role [16].

The corruption of projection data in X-ray CT with scattered radiation decreases low contrast detectability, reduces CT numbers and introduces cupping and streak artifacts in reconstructed images [4]. Scatter removal is also mandatory in X-ray CT imaging because of the need to have clinically acceptable low contrast detectability. The assessment of the scatter component in fan- and cone-beam $\mathrm{X}$-ray CT scanners is an active research area in quantitative imaging and there are many relevant contributions to this domain [5, 14, 39-44]. The most common technique used to reduce the detection of scattered radiation consists of using collimator plates inside the detector housing (septa) in multi-slice detector $\mathrm{CT}$ and antiscatter grids in flat-panel cone-beam CT scanners. The optimization of septa length and thickness (namely geometrical efficiency of detection system) as well as septa material is also being investigated through assessment of resulting scatter-to-primary ratio (SPR) using MC calculations. It has been shown that increasing the septa length could effectively reduce the contribution of scattered radiation, thus decreasing the SPR at the expense of additional manufacturing constraints to avoid possible septa plates' vibration during the gantry rotation [12]. It has long been recognized that the ideal research tool for scatter modeling and evaluation of scatter correction techniques is the MC method [18]. Given a known electronic density or attenuation distribution of the object, MC techniques allow detected events to be classified into unscattered and scattered events thus offering the possibility to determine directly the scatter component (which is often rather smooth). MC methods give further insight and might in themselves offer a possible correction procedure [43]. However, even with the use of acceleration techniques, these simulations require large amounts of computer time [45]. Moreover, the simulation of the scatter component for each patient is impractical in clinically feasible times. Faster implementations of hybrid approaches combining MC calculations and deterministic algorithms for the estimation of noise-free scatter projections while maintaining accuracy have also been described elsewhere [5, 46].

Analytical models for generation of transmission polyenergetic Poisson X-ray projections for testing image 
reconstruction and beam hardening correction algorithms can be used with some confidence for simple geometries and homogeneous objects; however, their application to more complex geometries and nonhomogeneous objects is complicated and prone to error. A more general and accurate approach for generation of data sets is to use MC simulations by paying special attention to the number of simulated events to reduce statistical uncertainties in the generated data sets.

\section{Development of computational models for use in Monte Carlo simulations}

Conceptually, the purpose of a physical phantom or computational model for X-ray CT modeling is to represent an organ or body region of interest, to allow modeling the chemical composition of the attenuating medium, which absorbs and scatters the X-ray beams in a manner similar to biological tissues [47]. In other terms, a physical phantom or mathematical model is generally designed to represent an organ or tissue of the body, an organ system, or the whole-body. Computerized anthropomorphic models can either be defined by mathematical (analytical) functions or digital (voxel-based) volume arrays [18]. Analytic models consist of regularly shaped continuous objects defined by combinations of simple mathematical geometries (e.g., right circular cylinders, spheres, or disks), whereas voxelbased models are mainly derived from segmented tomographic images of the human anatomy obtained by either $\mathrm{X}$-ray CT or magnetic resonance imaging (MRI). Any complex attenuating medium distribution can therefore be modeled without being confined to geometrical shapes. Analytical models, however, have the advantage of being able to model anatomical variability and dynamic organs easily. In addition, the disadvantage of the voxelized approach is that inherent errors are introduced due to the model voxelization. The discretization errors inherent in the voxelized representation may be reduced by finer sampling of the discretized models. More refined analysis of advantages and drawbacks of analytical versus voxelized model representation for simulation of imaging systems is described elsewhere [48].

\subsection{Stylized mathematical models}

The mathematical specifications for models that are available assume a specific age, height and weight. People, however, exhibit a variety of shapes and sizes. The first breakthrough in the use of MC techniques was the development of the Fisher-Snyder heterogeneous, hermaphrodite, anthropomorphic model of the human body in the 1970s [49]. This model consisted of spheres, ellipsoids, cones, tori and subsections of such objects, combined to approximate the geometry of the body and its internal structures. The representation of internal organs with this mathematical model is very crude, since the simple equations can only capture the most general description of an organ's position and geometry. In 1987, Cristy and Eckerman [50] of Oak Ridge National Laboratory (ORNL) developed a series of models representing children of different ages, one of which (the 15-year-old) also served as a model for the adult female.

Many stylized models have been developed specifically for assessment of image reconstruction techniques in X-ray CT and emission tomography and may not be suitable for other applications in radiological sciences (e.g., radiation dosimetry). Examples of these include the popular Shepp and Logan brain model [51], which has been used extensively during the early developments of image reconstruction methodologies and the FORBILD database developed by the Institute of Medical Physics of Erlangen University, Germany [52]. The latter family of objects comprises various organ models representing the head, abdomen, lung, thorax, hip and jaw, and are commonly used for evaluation of reconstruction algorithms, usually only with deterministic simulations.

Mathematical anthropomorphic models are continuously being improved. Recent three- and four-dimensional (space and time) computer models seek a compromise between ease of use, flexibility and the accurate modeling of populations of patient anatomies, and attenuation and scatter properties in patients. The use of dynamic anthropomorphic models in MC simulations is becoming possible, owing to the increasing availability of computing power. This includes the development of appropriate primitives that allow the accurate modeling of anatomical variations and patient motion, such as superquadrics [53] and nonuniform rational B-spline surfaces (NURBS) [54]. More recently, an efficient algorithm for the computation of X-ray transforms for superelipsoids and tori with monochromatic X-rays was developed to promote the use of superquadrics for CT simulation [55].

One such example is the 4D NURBS-based CardiacTorso (NCAT) model developed by Segars et al. [54], which brought several improvements to the earlier Mathematical CArdiac-Torso (MCAT) anthropomorphic model [56] that has been used extensively in emission computed tomography imaging research. The latter uses mathematical formulae, the size, shape and configurations of the major thoracic structures and organs such as the heart, liver, breasts, and rib cage to achieve realistic modeling. Incorporation of accurate models of cardiac and respiratory physiology into the current 4-D NCAT model was a significant step forward to account for inherent cardiac and respiratory motion not considered in the previous models. 
Fig. 3 Surface rendered images of the 4-D NCAT phantom showing from left to right: anterior, posterior and right lateral views with outer body surface removed to show the ribs cage and various organs modeled. Courtesy of Dr P. Segars [54]

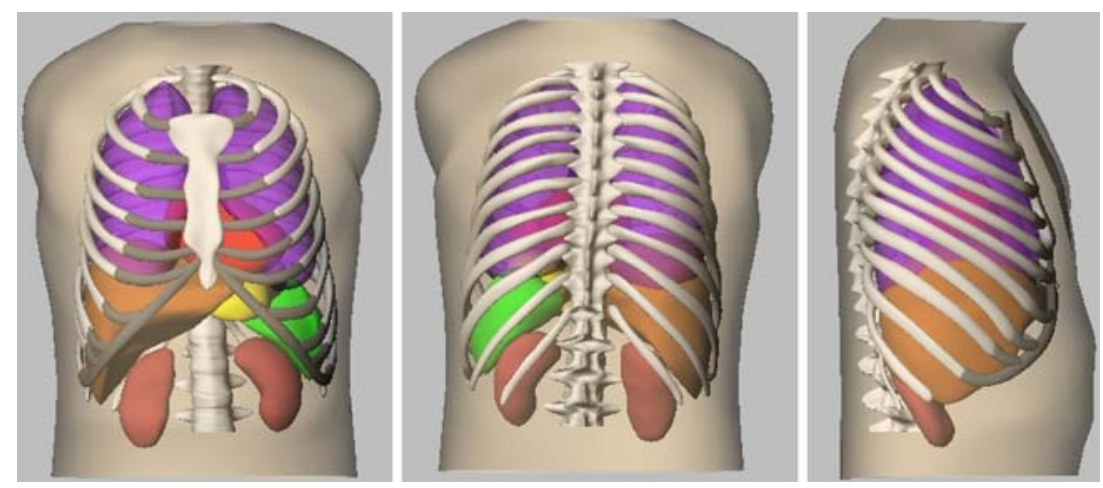

3D surface renderings illustrating anterior, posterior and right lateral views of the 4D NCAT model are shown in Fig. 3.

While much effort has been devoted towards the creation of human models, few research studies have been initiated to address the need for models supporting small animal imaging and dosimetry research. The recent interest in small animal imaging instrumentation for molecular imaging research has spurred the development of realistic computerized models modeling their anatomy and physiological functions. One such example is the 4D digital mouse model based on high-resolution 3D magnetic resonance microscopy acquired data and NURBS formalism mentioned above for modeling the organ shapes [57]. The NURBS primitives can elegantly model the complex organ shapes and structures, providing the foundation for a realistic model of the 3D mouse anatomy.

\subsection{Tomographic voxel-based models}

Modeling for imaging and dosimetry applications is best done with models that match the gross parameters of an individual patient. Anthropomorphic models with internally segmented structures make clinically realistic MC simulations possible. Zubal [58] developed a typical anthropomorphic voxel-based adult model by the manual segmentation of CT transverse slices of a living human male performed by medical experts. Each voxel of the volume contains an index number designating it as belonging to a given organ or internal structure. These indexes can then be used to assign a value, corresponding to, for example, electronic density. The same group has also developed a high resolution brain model based on an MRI scan of a human volunteer, which can be used for detailed investigations in the head. More recently, a new voxel-based whole body model, called VIP-Man [59] and a head/brain model [60] have been developed using high resolution transversal color photographic images obtained from the National Library of Medicine's visible human (VH) Project. A group at the National Research Center for Environment and Health (GSF) in Germany has also been developing some voxel-based models to cover persons of individual anatomy and includes at the moment two pediatric and five adult models of both sexes, different ages and stature, and several others are under construction [61]. Several national and international initiatives were also undertaken to develop Korean- and Chinese-type [62] equivalents to the above referenced $\mathrm{VH}$ project.

Despite the difficulties and ethical issues, the efforts devoted towards the development of voxel-based pediatric models and pregnant female are further emphasized in the construction of the two UF (University of Florida, USA) models (newborn female and 2-month-old male) [63] and the 30 weeks pregnant female tomographic model [64] opening the door to prospective imaging and dosimetry studies that would otherwise be difficult to perform.

\section{Acceleration of Monte Carlo simulations}

One significant problem in the use of MC calculations is the presence of statistical uncertainties (noise) in the estimates. A simple but not practical way to decrease statistical uncertainties is to run MC simulations for sufficiently long time (large number of histories) and use efficient variance reduction techniques. Currently, two approaches are used to reduce statistical uncertainties from MC calculations: hardware (parallelization) and software (de-noising and hybrid simulation) approaches. It should be noted that optimization and validation of these approaches is still an area of considerable research interest that requires further research and development efforts.

Parallel implementation of time-consuming MC calculations can be performed efficiently owing to the fact that particle histories are completely independent from each other [17]. A common way to parallelize MC codes is to put identical "clones" on the various processors; only the random number seeds are different [65]. However, special attention should be paid to seeds' initialization of random number generators (RNGs) for sequences to be uncorrelated. Another alternative would be to parallelize the RNGs used during simulation [66]. By spreading out the calcula- 
tions among many processors, a speed-up that approaches the number of processors being used could be attained. This can be achieved through the use of parallel processing environments including arrays of transputers, vector parallel supercomputers, massively parallel computers, a cluster of workstations in a local area network using a parallel computing simulator such as Parallel Virtual Machine (PVM), or geographically distributed platforms taking advantage of the latest developments in Grid technology. The latter requires substantial organizational skills and huge investments in physical resources (computers, disks and network) and relies on "grid middleware" software that guarantees the access and the coordinated use of the grid of networked computers (servers) for authorized users (clients) thus limiting its availability to big research centers.

A more convenient and less demanding approach to remove statistical fluctuations from noisy MC results is to use smoothing or de-noising algorithms. Some of the developed methods are inspired from classic image processing methods (e.g., filtering, anisotropic diffusion, wavelets, ...etc), whereas other methods do not have direct relationship with image processing techniques (e.g., Kawrakow's locally adaptive method, Fipple and Nusslin's formulation, ...etc) [67]. Although denoising seems to be the ideal solution to speed-up MC simulations, only one group reported on the use of dedicated curve fitting procedures inspired by the Richardson-Lucy deblurring algorithm to calculate scattered radiation projections in smallanimal cone-beam X-ray CT scanners [5]. It should be noted that most denoising techniques have been proposed with the aim of reducing MC statistical fluctuations for radiotherapy treatment planning. The optimization and implementation of similar techniques for $\mathrm{CT}$ simulation remains to be explored. Recently, El Naqa et al. [67] compared several denoising techniques including locally adaptive Savitzky-Golay filtering, content adaptive median hybrid filters, wavelet threshold denoising, anisotropic diffusion and noise reduction as an optimization problem. The results of denoising techniques effectiveness can be used for development of new denoising methods in the field of X-ray CT simulation.

Another approach to speed-up X-ray CT modeling is to use hybrid approaches combining MC and analytical simulations. In this approach, the contribution of the primary component to the projections is calculated through analytical simulations using ray-tracing methods whereas the contribution of the scatter component is calculated using pure MC simulations. The final projections are determined by appropriate combination of both simulation results. This approach has been used for modeling conventional radiographic imaging systems [25] and more recently for tomographic X-ray CT scanners [12] reporting good agreement between hybrid and pure MC simulated results
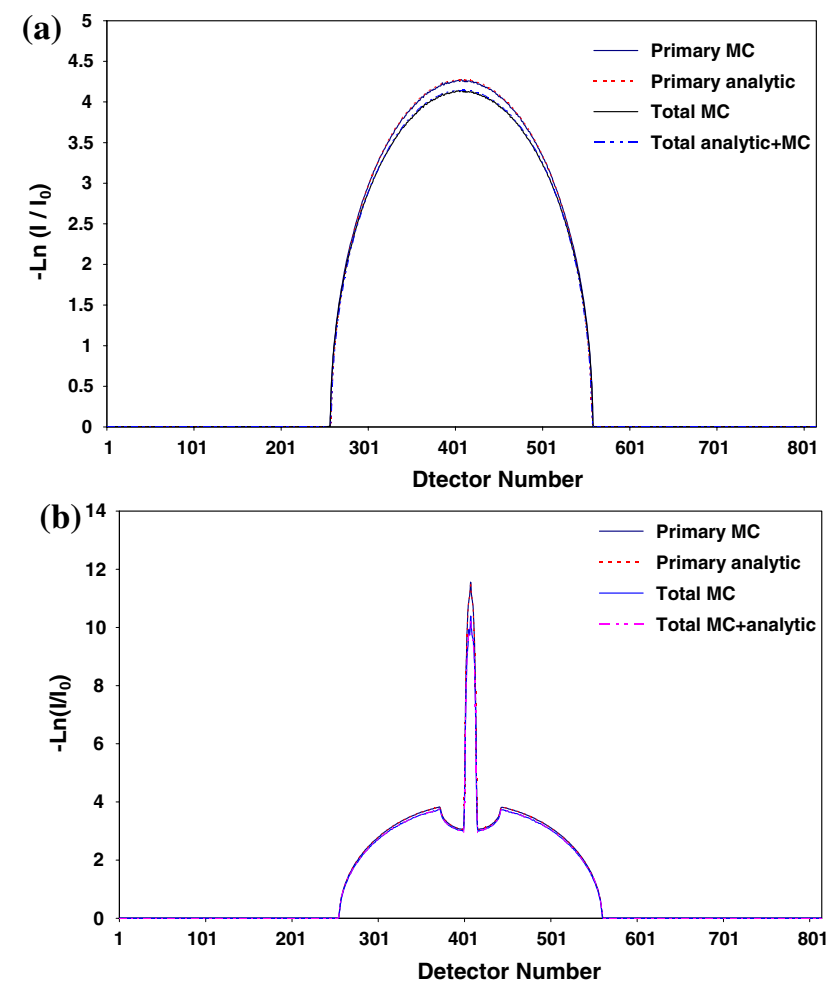

Fig. 4 Comparison of attenuation profiles for a uniform cylindrical water phantom (a) and water phantom containing centred steel rod insert (b) computed using pure MCNP4C-based Monte Carlo calculations and hybrid simulations combining analytical and Monte Carlo calculations

[44]. Figure 4 illustrates the excellent agreement between attenuation profiles of a uniform cylindrical water phantom and water phantom containing centred steel rod insert generated using pure Monte Carlo calculations based on our MCNP4C code and hybrid simulation combining analytical and Monte Carlo calculations.

\section{Summary}

Monte Carlo analysis in medical and biological engineering has been used for several decades, however with the ever-increasing power of desktop computers; the utility of Monte Carlo simulation is increasing. Today's applications of Monte Carlo techniques include: diagnostic imaging and radiation therapy, traffic flow, Dow-Jones forecasting, and oil well exploration, as well as more traditional physics applications like stellar evolution, reactor design, and quantum chromo-dynamics. Likewise, Monte Carlo methods are widely used in modelling of materials and chemicals, from grain growth modelling in metallic alloys, to behaviour of nanostrutures and polymers, and protein structure predictions. It should be noted that a few papers were published in this journal related to this specific topic, 
e.g., for parameter prediction in biomechanical models [68].

The use of the Monte Carlo method to simulate radiation transport has become the most accurate means of simulating medical imaging systems with the aim of optimizing instrumentation design or improving the accuracy of quantitative analysis and predicting absorbed dose distributions and other quantities of interest in diagnosis and radiation treatments of cancer patients. This trend has continued for the estimation of the absorbed dose in diagnostic procedures as well as the assessment of image quality and the quantitative accuracy of radiological imaging. As a consequence of this generalized use, many questions are being raised, primarily about the need and potential of Monte Carlo techniques, but also about how accurate they really are, what would it take to apply them clinically and make them available widely to the medical physics community at large. Many of these questions will be answered when Monte Carlo techniques are implemented and used in a reasonable amount of time using high powered computing workstations or distributed computing networks for more routine calculations and for in-depth investigations.

The combination of realistic computer models of the human anatomy and accurate models of the imaging process allows the simulation of X-ray CT data that are ever closer to actual patient data. Simulation techniques will find an increasingly important role in the future of medical imaging in light of the further development of realistic computer models, the accurate modeling of projection data and computer hardware. However, caution must be taken to avoid errors in the simulation process, and verification via comparison with experimental and patient data is essential.

Acknowledgments This work was supported by the Swiss National Science Foundation under grant SNSF 3100A0-116547.

\section{References}

1. Kalender WA (2006) X-ray computed tomography. Phys Med Biol 51:R29-43

2. Boone JM(2006) Multidetector CT: opportunities, challenges, and concerns associated with scanners with 64 or more detector rows. Radiology 241:334-337

3. Hsieh J (2003) Analytical models for multi-slice helical CT performance parameters. Med Phys 30:169-178

4. Tofts PS, Gore JC (1980) Some sources of artefact in computed tomography. Phys Med Biol 25:117-127

5. Colijn AP, Beekman FJ (2004) Accelerated simulation of cone beam X-ray scatter projections. IEEE Trans Med Imaging 23:584-590

6. DeMarco JJ, Cagnon CH, Cody DD, Stevens DM, McCollough $\mathrm{CH}$, Zankl $\mathrm{M}$ et al (2007) Estimating radiation doses from multidetector CT using Monte Carlo simulations: effects of different size voxelized patient models on magnitudes of organ and effective dose. Phys Med Biol 52:2583-2597
7. Lee SC, Kim HK, Chun IK, Cho MH, Lee SY (2003) A flat-panel detector based micro-CT system: performance evaluation for small-animal imaging. Phys Med Biol 48:4173-4185

8. Ning R, Tang X, Conover D (2004) X-ray scatter correction algorithm for cone beam CT imaging. Med Phys 31:1195-1202

9. Siewerdsen JH, Jaffray DA (2000) Optimization of X-ray imaging geometry (with specific application to flat-panel cone-beam computed tomography). Med Phys 27:1903-1914

10. Tu S-J, Shaw CC, Chen L (2006) Noise simulation in cone beam CT imaging with parallel computing. Phys Med Biol 51:12831297

11. Colijn AP, Zbijewski W, Sasov A, Beekman FJ (2004) Experimental validation of a rapid Monte Carlo based micro-CT simulator. Phys Med Biol 49:4321-4333

12. Ay M, Zaidi H (2005) Development and validation of MCNP4Cbased Monte Carlo simulator for fan- and cone-beam X-ray CT. Phys Med Biol 50:4863-4885

13. Kanamori H, Nakamori N, Inoue K, Takenaka E (1985) Effect of scattered X-ray on CT images. Phys Med Biol 30:239-249

14. Malusek A, Seger MM, Sandborg M, Alm Carlsson G (2005) Effect of scatter on reconstructed image quality in cone beam computed tomography: evaluation of a scatter-reduction optimisation function. Radiat Prot Dosimetry 114:337-340

15. Caon M, Bibbo G, Pattison J (1997) A comparison of radiation dose measured in CT dosimetry phantoms with calculations using EGS4 and voxel-based computational models. Phys Med Biol 42:219-29

16. Khodaverdi M, Chatziioannou AF, Weber S, Ziemons K, Halling H, Pietrzyk U (2005) Investigation of different micro-CT scanner configurations by GEANT4 simulations. IEEE Trans Nucl Sci 52:188-192

17. Andreo A (1991) Monte Carlo techniques in medical radiation physics. Phys Med Biol 36:861-920

18. Zaidi H (1999) Relevance of accurate Monte Carlo modeling in nuclear medical imaging. Med Phys 26:574-608

19. Rogers DWO (2006) Fifty years of Monte Carlo simulations for medical physics. Phys Med Biol 51:R287-R301

20. De Man B, Nuyts J, Dupont P, Marchal G, Suetens P (1999) Metal streak artifacts in X-ray computed tomography: a simulation study. IEEE Trans Nucl Sci 46:691-696

21. De Francesco D, Da Silva A. Multislice spiral CT simulator for dynamic cardiopulmonary studies. Medical imaging 2002: Physiology and function from multidimensional images, San Diego, 2002, 4683:305-316

22. DeMarco JJ, Cagnon CH, Cody DD, Stevens DM, McCollough CH, O'Daniel J et al (2005) A Monte Carlo based method to estimate radiation dose from multidetector CT (MDCT): cylindrical and anthropomorphic phantoms. Phys Med Biol 50:39894004

23. Boone JM, Buonocore MH, Cooper VN. (2000) Monte Carlo validation in diagnostic radiological imaging. Med Phys 27:1294-1304

24. Giersch J, Weidemann A, Anton G. (2003) ROSI-an object-oriented and parallel-computing Monte Carlo simulation for X-ray imaging. Nucl Instr Meth A 509:151-156

25. Winslow M, Xu XG, Yazici B. (2005) Development of a simulator for radiographic image optimization. Comput Methods Programs Biomed 78:179-190

26. Ay M, Shahriari M, Sarkar S, Adib M, Zaidi H. (2004) Monte Carlo simulation of X-ray spectra in diagnostic radiology and mammography using MCNP4C. Phys Med Biol 49:4897-4917

27. Tanner RJ, Chartier J-L, Siebert BRL, Agosteo S, Grosswendt B, Gualdrini $G$ et al (2004) Intercomparison on the usage of computational codes in radiation dosimetry. Radiat Prot Dosimetry 110:769-780 
28. Wiest PW, Locken JA, Heintz PH, Mettler FA Jr (2002) CT scanning: a major source of radiation exposure. Semin Ultrasound CT MR 23:402-410

29. Salvado M, Lopez M, Morant JJ, Calzado A. (2005) Monte Carlo calculation of radiation dose in CT examinations using phantom and patient tomographic models. Radiat Prot Dosimetry 114:364-368

30. Staton RJ, Lee C, Lee C, Williams MD, Hintenlang DE, Arreola MM, et al (2006) Organ and effective doses in newborn patients during helical multislice computed tomography examination. Phys Med Biol 51:5151-5166

31. Tzedakis A, Perisinakis K, Raissaki M, Damilakis J. (2006) The effect of $\mathrm{z}$ overscanning on radiation burden of pediatric patients undergoing head CT with multidetector scanners: a Monte Carlo study. Med Phys 33:2472-2478

32. Theocharopoulos N, Damilakis J, Perisinakis K, Tzedakis A, Karantanas A, Gourtsoyiannis N (2006) Estimation of effective doses to adult and pediatric patients from multislice computed tomography: a method based on energy imparted. Med Phys 33:3846-3856

33. Taschereau R, Chow PL, Chatziioannou AF (2006) Monte Carlo simulations of dose from microCT imaging procedures in a realistic mouse phantom. Med Phys 33:216-224

34. Mazonakis M, Tzedakis A, Damilakis J, Gourtsoyiannis N (2007) Thyroid dose from common head and neck CT examinations in children: is there an excess risk for thyroid cancer induction? Eur Radiol 17:1352-1357

35. LeHeron JC. CTDOSE (1993) A computer program to enable the calculation of organ dose, dose indices for CT examinations. Ministry of Health, National Radiation Laboratory, Christchurch

36. Jones DG, Shrimpton PC (1991) Survey of CT practice in the UK. Part 3: Normalised organ doses calculated using Monte Carlo techniques. NRPB-R250, Chilton

37. ImPACT. ImPACT: CT patient dosimetry calculator. London, 2006. Available at http://www.impactscan.org/ctdosimetry.htm

38. Ay M, Shahriari M, Sarkar S, Sardari D, Zaidi H (2005) Assessment of different computational models for generation of $\mathrm{X}$-ray spectra in diagnostic radiology and mammography. Med Phys 32:1660-1675

39. Chan HP, Doi K (1985) Physical characteristics of scattered radiation in diagnostic radiology: Monte Carlo simulation studies. Med Phys 12:152-165

40. Cheng CW, Taylor KW, Holloway AF (1995) The spectrum and angular distribution of X-rays scattered from a water phantom. Med Phys 22:1235-1245

41. Endo M, Tsunoo T, Nakamori N, Yoshida K (2001) Effect of scatter radiation on image noise in cone beam CT. Med Phys 28:469-474

42. Endo M, Mori S, Tsunoo T, Miyazaki H (2006) Magnitude and effects of X-ray scatter in a 256-slice CT scanner. Med Phys 33:3359-3368

43. Zbijewski W, Beekman FJ (2006) Efficient Monte Carlo-based scatter artifact reduction in cone-beam micro-CT. IEEE Trans Med Imaging 25:817-827

44. Ay M, Zaidi H (2006) Assessment of errors caused by X-ray scatter and use of contrast medium when using CT-based attenuation correction in PET. Eur J Nucl Med Mol Imaging 33:1301-1313

45. Schmidt B, Kalender WA (2003) Advanced method for calculating the scatter signal contribution in CT detectors by the Monte Carlo method. Z Med Phys 13:30-39

46. Kyriakou Y, Riedel T, Kalender WA (2006) Combining deterministic and Monte Carlo calculations for fast estimation of scatter intensities in CT. Phys Med Biol 51:4567-4586

47. Zaidi H, Xu XG (2007) Computational anthropomorphic models of the human anatomy: the path to realistic Monte Carlo modeling in medical imaging. Annu Rev Biomed Eng 9: doi:10.1146/annurev.bioeng.9.060906.151934
48. Goertzen AL, Beekman FJ, Cherry SR (2002) Effect of phantom voxelization in CT simulations. Med Phys 29:492-498

49. Snyder W, Ford MR, Warner G (1978) Estimates of specific absorbed fractions for photon sources uniformly distributed in various organs of a heterogeneous phantom. Pamphlet No. 5, revised. New York: Society of Nuclear Medicine

50. Cristy M, Eckerman KF (1987) Specific absorbed fractions of energy at various ages from internal photon sources. I Methods, II one year old, III five year old, IV ten year old, V fifteen year old male and adult female, VI new-born and VII adult male. ORNL/ TM 8381/V1-V7, Oak Ridge National Laboratory, Oak Ridge

51. Shepp LA, Logan BF (1974) The Fourier reconstruction of a head phantom. IEEE Trans Nucl Sci 21:21-43

52. Institute of Medical Physics, The FORBILD phantom database. 2006. Available at http://www.imp.uni-erlangen.de/phantoms/

53. Peter J, Gilland DR, Jaszczak RJ, Coleman RE. (1999) Fourdimensional superquadric-based cardiac phantom for Monte Carlo simulation of radiological imaging systems. IEEE Trans Nucl Sci 46:2211-2217

54. Segars WP (2001) Development, application of the new dynamic NURBS-based cardiac-torso (NCAT) phantom [Ph.D Thesis]. University of North Carolina, Chapel Hill

55. Zhu J, Zhao S, Ye Y, Wang G (2005) Computed tomography simulation with superquadrics. Med Phys 32:3136-3143

56. LaCroix K (1997) Evaluation of an attenuation compensation method with respect to defect detection in Tc-99m-sestamibi myocardial SPECT [Ph.D Thesis]. University of North Carolina, Chapel Hill

57. Segars WP, Tsui BM, Frey EC, Johnson GA, Berr SS (2004) Development of a 4D digital mouse phantom for molecular imaging research. Mol Imaging Biol 6:149-159

58. Zubal IG, Harrell CR, Smith EO, Rattner Z, Gindi G, Hoffer BP (1994) Computerized 3-dimensional segmented human anatomy. Med Phys 21:299-302

59. Xu XG, Chao TC, Bozkurt A (2000) VIP-Man: an image-based whole-body adult male model constructed from color photographs of the Visible Human Project for multi-particle Monte Carlo calculations. Health Phys 78:476-486

60. Chao T-c, Xu XG (2004) S-values calculated from a tomographic head/brain model for brain imaging. Phys Med Biol 49:49714984

61. Petoussi-Henss N, Zankl M, Fill U, Regulla D (2002) The GSF family of voxel phantoms. Phys Med Biol 47:89-106

62. Park JS, Chung MS, Hwang SB, Lee YS, Har DH, Park HS (2005) Visible Korean human: improved serially sectioned images of the entire body. IEEE Trans Med Imaging 24:352-360

63. Nipper JC, Williams JL, Bolch WE (2002) Creation of two tomographic voxel models of paediatric patients in the first year of life. Phys Med Biol 47:3143-3164

64. Shi CY, Xu XG (2004) Development of a 30-week-pregnant female tomographic model from computed tomography (CT) images for Monte Carlo organ dose calculations. Med Phys 31:2491-2497

65. Zaidi H, Labbé C, Morel C (1998) Implementation of an environment for Monte Carlo simulation of fully 3D positron tomography on a high-performance parallel platform. Parallel Comput 24:1523-1536

66. Maigne L, Hill D, Calvat P, Breton V, Reuillon R, Lazaro D et al (2004) Parallelization of Monte Carlo simulations and submission to a Grid environment. Parallel Proc Lett 14:177-196

67. El Naqa I, Kawrakow I, Fippel M, Siebers JV, Lindsay PE, Wickerhauser MV et al (2005) A comparison of Monte Carlo dose calculation denoising techniques. Phys Med Biol 50:909-922

68. Hughes RE, An KN (1997) Monte Carlo simulation of a planar shoulder model. Med Biol Eng Comput 35:544-548 